Service social

\title{
Partis politiques québécois, personnes âgées et promesses d'interventions gouvernementales
}

\section{Réjean Landry}

Volume 34, numéro 1, 1985

Personnes âgées, milieux de vie et pratiques sociales

URI : https://id.erudit.org/iderudit/706254ar

DOI : https://doi.org/10.7202/706254ar

Aller au sommaire du numéro

Éditeur(s)

École de service social de l'Université Laval

ISSN

1708-1734 (numérique)

Découvrir la revue

Citer cet article

Landry, R. (1985). Partis politiques québécois, personnes âgées et promesses

d'interventions gouvernementales. Service social, 34(1), 107-129.

https://doi.org/10.7202/706254ar d'utilisation que vous pouvez consulter en ligne.

https://apropos.erudit.org/fr/usagers/politique-dutilisation/ 


\section{Partis politiques québécois, personnes âgées et promesses d'interventions gouvernementales *}

\section{Réjean Landry}

Des facteurs d'ordre électoral, économique et psychosocial devraient inciter les partis politiques à promettre des interventions gouvernementales comportant des bénéfices spécifiques aux personnes âgées (Austin et Loeb, 1982; Cook, 1982; Kutza et Zweibel, 1982). L'analyse du contenu des programmes des partis politiques du Québec, depuis 1960, révèle toutefois que cette population constitue pour eux une cible peu importante. Cet état de fait pourrait s'expliquer par le caractère très hétérogène de cette population et par le caractère inapproprié de l'âge comme critère d'éligibilité aux bénéfices des interventions gouvernementales. Ce dernier facteur expliquerait d'ailleurs la place restreinte qu'occupe cette population dans les programmes des partis uniquement si les coûts de production des interventions gouvernementales sont élevés et si les bénéfices offerts aux personnes âgées sont tangibles plutôt que symboliques.

Le but de cet article est de démontrer que ce caractère inapproprié de l'âge comme critère n'explique pas convenablement la place modeste des personnes âgées dans les programmes des partis parce que les bénéfices promis sont généralement peu tangibles et que les partis font très rarement état des coûts de leurs promesses. La première partie de l'article traite de la place des programmes des partis dans le processus démocratique ; la seconde partie expose la méthode utilisée pour analyser le contenu des promesses d'action, tandis que la troisième présente les résultats de l'analyse des caractéristiques des coûts et bénéfices conséquents. 


\section{L'offre de promesses d'interventions gouvernementales}

Les résultats d'une élection ne dépendent pas uniquement des programmes des partis car les individus arrivent à prendre une décision en se fondant simultanément sur la loyauté envers un parti et le contenu des programmes. Il existe une sorte de fusion inévitable entre ces deux facteurs qui fait qu'on ne peut choisir isolément l'un ou l'autre. La compétition pour l'obtention des votes fait que les partis en viennent à être identifiés à des actions particulières. Leurs engagements les plus fermes sont consignés dans leurs programmes. Ces documents constituent les seuls énoncés clairs que les électrices et les électeurs peuvent leur attribuer. Même si peu de personnes en prennent connaissance, ils constituent les sources auxquelles se réfèrent les journalistes et les politiciens (Robertson, 1976: 72). Ils influencent les décisions de l'électorat de deux façons (Pomper, 1980: 129): ils fournissent un moyen pour établir un lien entre un parti et un programme en permettant aux individus de porter un jugement sur les performances passées, et les bénéfices qu'il promet pour l'avenir, et, d'autre part, ils constituent des engagements à accomplir des actions spécifiques, ce qui fait que le vote devient autant un choix de politique que de parti. Enfin, s'ils revêtaient aussi peu de signification que certains le prétendent (Ostrogorski, 1964, vol. 2 : 138-139), pourquoi donc observerait-on autant de conflits internes au moment des congrès des partis ? Les congrès récents du Parti libéral du Québec (P.L.Q.) et du Parti québécois (P.Q.) tendent à montrer que les membres et les leaders prennent très au sérieux le contenu de leurs programmes officiels.

Cette perspective se fonde sur l'existence d'une double rationalité : celle des électeurs et celle des dirigeants des partis. Les programmes permettent aux premiers de choisir celui dont les interventions promises maximisent leurs utilités (unités de bénéfices monétaires et non monétaires). D'autre part, pour les mêmes raisons, les partis peuvent augmenter les votes en modifiant le contenu de leurs programmes (Downs, 1957 ; Hayes, 1981).

Les différences observables d'un parti à l'autre résultent de l'état d'incertitude qui pèse sur les choix : les dirigeants ne connaissent pas parfaitement les interventions gouvernementales qui maximisent les votes alors que les électrices et les électeurs ne connaissent pas parfaitement les bénéfices découlant des interventions promises. En conséquence, les partis peuvent offrir des interventions différentes, espérant avoir jaugé de façon correcte les demandes de l'électorat. 
Qu'en est-il des promesses des programmes qui visent explicitement les personnes âgées ? Nous avons tenté d'évaluer les bénéfices et les coûts des promesses adressées à cette population en effectuant une analyse du contenu des programmes des partis politiques du Québec couvrant la période 1960-1981.

\section{La méthode de collecte des données}

La méthodologie définit les règles du jeu de l'activité scientifique et celles-ci dépendent de l'objectif de l'étude à réaliser. L'analyse de contenu comporte des règles fiables et valides pour effectuer la présente recherche.

D'après Holsti (1969: 14), "L'analyse de contenu regroupe toutes les techniques permettant de faire des inférences en identifiant objectivement et systématiquement certaines caractéristiques des messages". Elle peut prendre la forme d'une analyse qualitative ou quantitative :

"L'analyse classique est qualitative : elle ne procède à aucune mesure, à aucune quantification. Son souci est de rechercher la signification du document, aussi bien la signification évidente que la signification implicite. [...] L'analyse quantitative prétend aboutir à des analyses par le recours à la quantification. Il s'agit toujours d'interpréter les documents, mais on espère arriver grâce à cette technique à une analyse objective, indépendante de la personnalité de l'analyste et de ses jugements.» (Del Bayle, 1978 : 111-112.)

Une analyse de contenu doit identifier les données à analyser, la façon de les définir et la population d'où celles-ci originent. Cette méthode est ici utilisée dans une perspective d'étude évaluative parce que les caractéristiques des coûts et des bénéfices des promesses d'interventions gouvernementales sont comparées selon des périodes et des partis. Le devis méthodologique de l'étude proposée sert à tester des hypothèses. (Graphique 1.) 


\section{GRAPHIQUE 1}

\section{Devis d'analyse de contenu visant à tester des hypothèses}

La réalisation d'une analyse de contenu utilise toutefois un devis plus détaillé comprenant cinq opérations principales :

1) la sélection de l'unité d'analyse;

2) la sélection des documents à analyser ;

3) l'enregistrement des données ;

4) la vérification des données ;

5) l'analyse des données.

\section{Sélection de l'unité d'analyse}

L'unité d'analyse retenue ici est la proposition d'intervention gouvernementale qui est un énoncé comprenant quatre éléments :

- un destinateur: Qui énonce une intervention? (ex. : le P.Q.)

- un verbe connecteur: le verbe d'action utilisé par le destinateur (ex. : promet)

- I'attribut d'un objet: Quoi? Quel est l'attribut de l'objet de l'intervention offerte par le destinateur (ex.: une politique de rénovation des logements pour permettre le maintien des retraités dans un logement décent)

- un destinataire : $A$ qui s'adresse l'intervention énoncée par le destinateur? (ex. : retraités). 


\section{Sélection des documents à analyser}

L'étude proposée évalue les caractéristiques des coûts et des bénéfices des interventions gouvernementales contenues dans les programmes d'action des partis politiques du Québec de 1960 à 1981 (tableau 1).

TABLEAU 1

Programmes retenus pour analyse

\begin{tabular}{|l|ccccccc|}
\hline Partis & \multicolumn{7}{|c|}{ Années } \\
& 1960 & 1962 & 1966 & 1970 & 1973 & 1976 & 1981 \\
\hline P.L.Q. & $\mathrm{X}$ & $\mathrm{X}$ & $\mathrm{X}$ & $\mathrm{X}$ & $\mathrm{X}$ & $\mathrm{X}$ & $\mathrm{X}$ \\
U.N. & $\mathrm{X}$ & $\mathrm{X}$ & $\mathrm{X}$ & $\mathrm{X}$ & $\mathrm{X}$ & $\mathrm{X}$ & $\mathrm{X}$ \\
P.Q. & & & & $\mathrm{X}$ & $\mathrm{X}$ & $\mathrm{X}$ & $\mathrm{X}$ \\
\hline
\end{tabular}

\section{Enregistrement des données}

La fiche d'analyse de contenu et le document définissant son utilisation définissent les règles relatives à l'enregistrement des données (Landry, 1983). Cette fiche et le guide d'utilisation comprennent quatre sections :

- identification de la proposition étudiée ;

- grille de classification de l'objet de la proposition d'intervention selon les catégories utilisées dans le système : Plan, Programme, Budget du gouvernement du Québec ;

- grille de qualification des caractéristiques des coûts et des bénéfices de la proposition d'intervention suivant les concepts de la théorie des choix collectifs (contenu monétaire des coûts et bénéfices, difficulté de mesurer les coûts et bénéfices, degré d'exclusion et de concentration des bénéfices);

- texte de la proposition.

La fiche d'analyse de contenu constitue une simulation d'entrevues réalisées à l'aide d'un questionnaire fermé (Krippendorff, 1980). L'analyste pose des questions à des documents et répond à un questionnaire à l'aide des instructions fournies dans le guide d'utilisation de cette fiche. 


\section{Vérification des données}

La vérification des données renvoie à l'étape où l'on corrige les erreurs attribuables à l'analyse de contenu ou à la retranscription des données. L'analyste prend en note tous les cas pour lesquels la codification des réponses soulève des doutes ou des difficultés particulières. Ces cas sont réglés lors de réunions hebdomadaires avec le responsable de l'équipe. Les décisions prises à ce moment visent à accroître la fiabilité de la codification en précisant, s'il y a lieu, les instructions de la codification de la fiche d'analyse de contenu. D'autre part, un programme informatique de vérification (écrit en langage SAS) a été conçu pour produire les informations suivantes:

- le texte de chaque proposition d'intervention classée dans chacun des éléments de la grille de classification et de la grille de qualification;

- le texte des propositions pour les cas où des réponses sont logiquement incompatibles.

Les informations produites par l'ordinateur à cette étape sont ensuite vérifiées manuellement et corrigées sur le ruban d'ordinateur. Ces listes constituent aussi des catalogues auxquels on peut se référer par la suite pour s'assurer que les propositions ayant les mêmes caractéristiques sont toujours classées de la même façon.

\section{Analyse des résultats}

Trois raisons principales incitent les partis politiques à faire des promesses qui comporteraient des bénéfices spécifiques aux personnes âgées (Austin et Loeb, 1982; Cook, 1982; Kutza et Zweibel, 1982). La première raison est d'ordre strictement électoral : l'accroissement de la proportion des personnes âgées au sein de l'ensemble de la population électorale a fait un bond considérable depuis une vingtaine d'années. Ainsi, le nombre de personnes de 65 ans et plus est passé de 296000 à 546000 personnes de 1960 à 1980, soit un bond de 5,8\% à 8,6\% de la population québécoise totale. Au même moment, leur poids électoral s'est élevé de $9,7 \%$ à $11,9 \%$. Au recensement de 1971 , la moitié des circonscriptions comprenait de $8 \%$ à $11,9 \%$ d'électeurs âgés, un quart en comptait moins de $8 \%$, tandis que l'autre quart dépassait $12 \%$ (Crête et Landry, 1983). Leur poids électoral potentiel est en voie de devenir déterminant dans bon nombre de circonscriptions où la marge entre le candidat élu et le candidat défait est inférieure à $15 \%$. 
D'autre part, les partis politiques peuvent aussi justifier leurs promesses d'interventions à l'avantage des personnes âgées en s'appuyant sur des arguments à caractère économique. Les opportunités d'emplois se font de plus en plus rares à mesure que les travailleurs avancent en âge, réduisant ainsi leurs possibilités de maintenir leur survie économique de façon autonome. De plus, la retraite entraîne une diminution de revenus qui ne s'accompagne pas nécessairement d'une réduction comparable des dépenses. Enfin, la population âgée est très souvent considérée comme une catégorie présumément pauvre et avec un mauvais état de santé. La sympathie qu'attire leur sort dans les autres couches de la population justifie donc les partis politiques de promettre des interventions visant à l'améliorer.

En troisième lieu, l'incitation serait d'ordre psychosociologique. En effet, les partis politiques peuvent estimer qu'il est électoralement rentable de promettre des mesures de compensation qui visent à redresser le tort causé aux personnes âgées par des mesures gouvernementales concernant l'âge de la retraite, l'accès aux bénéfices de programmes tels que l'assurance-chômage, etc. Les préjudices causés par certains programmes gouvernementaux seraient ainsi compensés par les bénéfices que leur procurent d'autres mesures publiques. Ce principe de compensation se justifie d'autant plus facilement que tous reconnaissent la contribution qu'elles ont apporté à l'ensemble de la société. Chaque génération de citoyens accepte de supporter ces coûts en faisant l'hypothèse que les générations futures accepteront de supporter les coûts des bénéfices accordés aux générations précédentes.

Ces arguments (non mutuellement exclusifs) d'ordre électoral, économique et psychosociologique permettent de prédire qu'un parti politique qui cherche à maximiser ses appuis électoraux tirera avantage à augmenter la proportion de ses promesses d'interventions gouvernementales dont les bénéfices visent spécifiquement les personnes âgées. Qu'en est-il exactement?

La place que les personnes âgées occupent dans les programmes des partis, même si elle s'accroît lentement, est bien loin de correspondre à l'importance de leur poids dans le corps électoral : pour la période allant de 1960 à 1981 (sept élections), ils contiennent seulement cent quarante-et-une propositions en ce sens. Les partis ont présenté 16 (11\%) de ces promesses dans leurs programmes de 1960, 1962, 1966 et 1970 tandis que leurs seuls programmes de 1981 en totalisent 85 , soit $60 \%$ de toutes les promesses d'interventions s'adressant spécifiquement aux personnes âgées. Au mieux, la proportion serait passée de $1 \%$ à $2 \%$ en 1960 à environ 4\% en 1981. Le poids des personnes âgées dans les 
programmes des partis représente donc moins du tiers du poids qu'elles ont dans le corps électoral. Par ailleurs, la population adulte (18 à 59 ans) reçoit en moyenne $40 \%$ des promesses d'interventions au cours des années soixante, tandis que celles qui ne comportent pas suffisamment d'informations pour être classées suivant des cibles d'âge en totalisent un peu plus de $50 \%$.

Le programme du Parti québécois est celui qui en offre en moyenne le plus grand nombre: de 1970 à 1981, l'Union nationale (U.N.) offre 2.2 propositions d'interventions par programme, le P.L.Q. 7.4 et le P.Q. 18.5. Afin d'éliminer de la série chronologique les effets dépendant d'une seule élection, on a tenté d'estimer la tendance lissée, i.e. propre à chaque parti, en calculant la moyenne de trois programmes avec deux programmes adjacents. Ces moyennes móbiles indiquent une tendance, par exemple à l'augmentation ou à la diminution, voire à la constance. La tendance à l'augmentation est beaucoup plus forte chez les péquistes que chez les libéraux (Tableau 2). Les déviations les plus grandes se produisent en 1973 dans le cas du P.L.Q. et en 1976 dans le cas du P.Q. Leur ampleur indique une rupture confirmée par l'accroissement considérable de ce nombre de propositions. L'Union nationale se distingue des autres par son plus petit nombre de propositions ainsi que par sa plus faible tendance à en augmenter le nombre.

En dépit de la forte tendance du P.L.Q. et du P.Q. à augmenter le nombre de ces promesses, il faut noter que les personnes âgées représentent une cible beaucoup plus marginale que ne l'indique leur poids démographique et électoral. Pourquoi donc? Ne constituentelles pas une cible importante dans les programmes des partis politiques?

Les partis sont probablement moins incités qu'on le pense généralement à considérer la population âgée en tant que cible parce que même si elle peut être influencée par les bénéfices promis sur des enjeux spécifiques, il est loin d'être certain qu'elle constitue un bloc de votes homogènes (Hudson et Binstock, dans: Binstock et Shanas, Handbook, 1976; Kutza et Zweibel, 1982 : 58). Elle constituerait, en fait, un ensemble fort diversifié de groupes que les partis ont de la difficulté à identifier avec suffisamment de précision pour leur promettre plus d'interventions bénéfiques. Il s'agit vraisemblablement d'une cible mouvante dont l'importance électorale varie en fonction de contextes historiques spécifiques. Trois types de changements compliquent l'identification de cette cible : le passage du marché du travail à la retraite, l'allongement de l'espérance de vie et, finalement, l'amélioration de la qualité de la santé des personnes âgées. Les changements qui surviennent relativement à ces trois facteurs permettent de distinguer au moins 
TABLEAU 2

Propositions visant les personnes âgées, moyenne de trois programmes, et déviations de la moyenne

\begin{tabular}{|c|ccc|ccc|ccc|ccc|}
\hline & \multicolumn{3}{|c}{ P.L.Q. } & \multicolumn{3}{c|}{ U.N. } & \multicolumn{3}{c|}{ P.Q. } & \multicolumn{3}{c|}{ Total } \\
& $\mathbf{n}$ & $\mathbf{m}$ & $\mathbf{d}$ & $\mathbf{n}$ & $\mathbf{m}$ & $\mathbf{d}$ & $\mathbf{n}$ & $\mathbf{m}$ & $\mathbf{d}$ & $\mathbf{n}$ & $\mathbf{m}$ & $\mathbf{d}$ \\
\hline 1960 & 4 & & & 0 & & & - & - & - & 4 & \\
1962 & 0 & 2.6 & +2.6 & 1 & .6 & -.4 & - & - & - & 1 & 3.3 & +2.3 \\
1966 & 4 & 2.3 & -1.7 & 1 & 1 & 0 & - & - & - & 5 & 3.6 & -1.4 \\
1970 & 3 & 3.3 & +.3 & 1 & 1.6 & +.6 & 1 & - & - & 5 & 7 & +2 \\
1973 & 3 & 6 & +3 & 3 & 2.6 & -.4 & 5 & 6.6 & +1.6 & 11 & 15.3 & +4.3 \\
1976 & 12 & 13.6 & +1.6 & 4 & 4.3 & +.3 & 14 & 24 & +10 & 30 & 42 & +12 \\
1981 & 26 & & & 6 & & & 53 & & & 85 & & \\
\hline $\bar{x}$ & 7.4 & & & 2.2 & & & 18.5 & & & 20.1 & \\
\hline
\end{tabular}

$\mathrm{n}=$ nombre de propositions par programme

$m=$ moyenne de trois programmes avec deux programmes adjacents

$\mathrm{d}=$ déviations de $\mathrm{m}$

$\bar{x}=$ moyenne des $n$ 
quatre sous-populations distinctes : les travailleurs âgés (55-64 ans); les jeunes-vieux (65-74 ans); les vieux d'âge moyen (75-84 ans); et finalement ceux de 85 ans et plus. Les problèmes propres à chaque groupe varient suffisamment pour compliquer l'élaboration de ces sous-populations. La diversité des situations que recouvrent ces quatre souspopulations tend à montrer que l'âge ne constitue pas la cible parfaite sur laquelle les partis pourraient centrer les bénéfices découlant de leurs promesses d'intervention.

À lui seul, le critère d'âge ne serait donc pas un critère d'éligibilité approprié pour définir l'accès aux bénéfices des interventions gouvernementales. Les partis seraient incités à utiliser le critère de l'âge comme un outil de rationnement dans le seul but de limiter les coûts; il constituerait donc un moyen de restreindre l'offre de bénéfices et de services publics à une bande étroite de bénéficiaires.

Par ailleurs, une promesse d'intervention gouvernementale fondée sur ce critère soulève deux types de difficultés. Elle peut créer des problèmes d'équité parce qu'elle peut être à la fois trop inclusive et trop exclusive. À supposer qu'elle vise tous les membres d'un groupe d'âge, elle rate partiellement sa cible si son objectif est de fournir des services aux membres dont les besoins sont les plus aigus. C'est le cas où les millionnaires reçoivent leur chèque du régime canadien des pensions de vieillesse même s'ils n'en ont pas besoin pour leur survie économique. D'autre part, l'âge constitue un critère trop exclusif, l'omission d'un bénéfice fondé sur le critère de l'âge ne pouvant se justifier qu'en autant qu'il soit possible de démontrer qu'il existe un lien étroit entre l'âge et des attributs spécifiques des interventions gouvernementales. Ce critère en devient un de ségrégation et de discrimination dans toutes les situations pour lesquelles cette démonstration ne peut être établie. L'exemple typique est la personne de 64 ans qui est sans travail et sans assurance-chômage - et qui n'est pas "millionnaire» - qui doit attendre d'avoir atteint le seuil de ses 65 ans pour être éligible aux pensions de vieillesse du gouvernement canadien. Les partis politiques peuvent donc, dans une certaine mesure, se montrer réticents à promettre des interventions gouvernementales dont l'accès aux bénéfices est fondé uniquement sur le critère de l'âge en raison de l'arbitraire de ce critère d'éligibilité.

Ces raisons n'expliquent toutefois pas convenablement la place restreinte qu'occupent les promesses d'action faites aux personnes âgées dans les programmes des partis politiques:

- la nécessité de rationner l'offre de bénéfices en utilisant le critère de l'âge constitue une explication plausible uniquement si le coût de production des interventions promises est élevé ; 
- l'arbitraire de l'âge comme critère d'exclusion constitue une explication plausible uniquement si celles-ci comportent des bénéfices tangibles.

Le bien-fondé de ces explications sera maintenant considéré plus en détail en examinant l'objet des promesses d'action ainsi que les caractéristiques des coûts et des bénéfices qui y sont associés.

\section{L'objet des promesses d'action}

Les promesses d'interventions gouvernementales contenues dans les programmes des partis ont été classifiées suivant les catégories du système P.P.B. (plan-programme-budget) utilisé par le gouvernement du Québec. Les propositions d'intervention s'adressant spécifiquement aux personnes âgées concernent douze objets différents bien que, comme le tableau 3 l'indique, les trois quarts de celles-ci concernent trois questions : la santé et les services sociaux (40); la sécurité sociale (39) et les conditions de travail (29). Si l'on ajoute les propositions concernant le logement (9) et celles relatives aux services publics (8), on constate que celles qui touchent ces cinq types d'objets totalisent $88 \%$ de toutes les promesses.

Avant d'aller plus avant, il peut être utile d'illustrer concrètement, à l'aide de quelques exemples, le contenu des propositions d'interventions :

1960 : Le P.L.Q. versera une allocation supplémentaire de $10 \$$ par mois aux bénéficiaires de pensions de vieillesse, à partir de 70 ans, et selon leurs besoins.

1966 : Le P.L.Q. promet, dès 1966, la construction de logements à loyer modique qui seront destinés en priorité aux couples âgés.

1970: Le P.L.Q. propose une véritable politique des loisirs qui doit être orientée de façon coordonnée en fonction des besoins des personnes âgées.

1973 : Le P.Q. permettra au travailleur, selon ses propres désirs, de prendre sa retraite à partir de 55 ans, ou de travailler jusqu'à un âge avancé.

1976 : Le P.L.Q. s'engage à rendre justice aux personnes âgées depuis trop longtemps oubliées par notre société.

1981 : Le P.L.Q. modifiera la loi des régimes supplémentaires de rentes pour exiger que la rente soit reversée au conjoint survivant.

1981: Le P.Q. promet une politique de rénovation des logements pour permettre le maintien des retraités dans un logement décent. 
TABLEAU 3

Objets des promesses d'interventions gouvernementales, par parti, 1960-1981

\begin{tabular}{|c|c|c|c|c|c|c|c|c|c|c|c|c|c|c|c|c|}
\hline \multirow{2}{*}{\begin{tabular}{|l} 
Sécurité sociale \\
\end{tabular}} & $\begin{array}{c}1960 \\
\text { P.L.Q. U.N. }\end{array}$ & $\begin{array}{c}1962 \\
\text { P.L.Q. U.N. }\end{array}$ & $\begin{array}{c}1966 \\
\text { P.L.Q. U.N. }\end{array}$ & \multicolumn{2}{|c|}{$\begin{array}{r}1970 \\
\text { P.L.Q. U.N. }\end{array}$} & \multirow{2}{*}{\begin{tabular}{c|} 
P.Q. \\
1
\end{tabular}} & \multicolumn{3}{|c|}{$\begin{array}{c}1973 \\
\text { P.L.Q. U.N. P.Q. }\end{array}$} & \multicolumn{3}{|c|}{$\begin{array}{c}1976 \\
\text { P.L.Q. U.N. P.Q. }\end{array}$} & \multicolumn{3}{|c|}{$\begin{array}{c}1981 \\
\text { P.L.Q. U.N. P.Q. }\end{array}$} & \multirow{2}{*}{$\frac{\text { Total }}{39}$} \\
\hline & 2 & & $\begin{array}{ll}2 & 1\end{array}$ & & & & 1 & 2 & 3 & 1 & 1 & 2 & 5 & 4 & 14 & \\
\hline Santé et services sociaux & & & 1 & 1 & & & 1 & & & 5 & 2 & 4 & 11 & & 15 & 40 \\
\hline Logement & & & 1 & & & & & & & 1 & & 2 & & 1 & 4 & 9 \\
\hline Conditions de travail & 1 & 1 & & 1 & & & & 1 & 2 & & 1 & 3 & 4 & & 14 & 29 \\
\hline Condition féminine & 1 & & & & & & & & & & & & & & & 1 \\
\hline Loisirs et sports & & & & 1 & & & & & & 1 & & & 1 & & & 3 \\
\hline Services publics & & & & & & & 1 & & & 2 & & 3 & & 1 & 1 & 8 \\
\hline Fiscalité & & & & & & & & & & 1 & & & & & 1 & 2 \\
\hline Éducation & & & & & & & & & & 1 & & & 1 & & 2 & 4 \\
\hline Droits et libertés & & & & & & & & & & & & & 3 & & & 3 \\
\hline Politique et recherche & . & & & & & & & & & & & & 1 & & 1 & 2 \\
\hline Constitution & & & & & & & & & & & & & & & 1 & 1 \\
\hline Total & 0 & 1 & 4 & 3 & 1 & 1 & 3 & 3 & 5 & 12 & 4 & 14 & 26 & 6 & 53 & 141 \\
\hline
\end{tabular}


Les partis se distinguent les uns des autres d'une façon particulièrement évidente sur le thème des conditions de travail ainsi que sur celui des droits et libertés de la personne. Si le P.Q. s'avère le champion dans le domaine des conditions de travail promises aux travailleurs âgés, le P.L.Q. se démarque en étant le seul à faire des promesses visant à assurer les droits et libertés des personnes âgées.

Bien que le nombre de promesses adressées aux personnes âgées ne soit pas très élevé, il ressort clairement que la sécurité sociale constitue le seul thème ayant fait l'objet de promesses au cours de toute la période étudiée. D'autre part, même si plus de $80 \%$ des propositions d'interventions concernant explicitement ce groupe ont été formulées pour les campagnes électorales de 1976 et 1981, on voit émerger le thème de la santé et des services sociaux à partir de 1966, et, à partir de 1973, des conditions de travail et des services publics.

Les travaux de Guillemard $(1983,1984)$ et Zay (1984) sur les interventions accomplies par l'État notent une évolution semblable à celle des promesses. D'après Guillemard, les interventions de l'État français dans le domaine de la vieillesse s'articulent autour de trois axes principaux: la retraite, le mode de vie et l'emploi. Et, selon elle, l'évolution se décompose suivant trois périodes : la première, qui va de 1944 au début des années 1960, est dominée par des actions d'assistance qui visent à suppléer les carences des régimes de retraite qui se mettent en place. La seconde, qui s'étend approximativement de 1960 à 1975, touche le mode de vie par l'action de l'État pour la réinsertion sociale de la population âgée et par la promotion de mesures concernant notamment le logement et l'équipement sanitaire. À partir de 1975, deux thèmes dominent : d'une part, les interventions de l'État à l'égard de l'insertion sociale se réduisent de plus en plus à des mesures de maintien à domicile; d'autre part, la crise et ses conséquences sur l'emploi le forcent à prendre des mesures relativement à l'emploi des travailleurs âgés.

Pour sa part, Zay (1984: 104) synthétise le discours et les interventions québécoises des quinze dernières années en formulant cinq propositions :

— «changer la mentalité de la société québécoise à l'égard de la vieillesse et du vieillissement";

- "améliorer le revenu de remplacement des aînés au moment de leur entrée dans le soi-disant "vieil âge"";

- "accroître les possibilités de choix quant au moment de la prise de la retraite";

— "faciliter à chacun de vivre dans le logement de son choix»";

- "favoriser l'autonomie et l'indépendance de la population âgée». 
L'examen des objets sur lesquels portent les promesses d'interventions gouvernementales ne peut toutefois pas expliquer la place restreinte qu'occupent les personnes âgées dans les programmes des partis politiques. Cet objectif peut être atteint, notamment, en examinant les caractéristiques des bénéfices et des coûts associés aux promesses d'action.

\section{a) Bénéfices et coûts monétaires}

La place restreinte que détiennent les personnes âgées dans les programmes des partis s'explique-t-elle par les bénéfices et les coûts monétaires qui découlent des promesses d'action qui les visent de façon explicite? Les données recueillies dans notre étude montrent que seulement 14 des 141 promesses d'intervention s'adressant à elles depuis 1960 comportent des bénéfices monétaires, c'est-à-dire comprenant des informations à propos d'une unité de mesure (en fait, des dollars) et une quantité (par exemple : 100). D'autre part, les partis ont promis de leur donner de l'argent sans mentionner de montant, ce que l'on appelle des bénéfices non explicites, à 46 reprises depuis 1960 , tandis que le reste, soit 90 , ne contient aucune information à ce niveau. Par ailleurs, les données indiquent que le P.Q. a plus tendance que le P.L.Q. et l'U.N. à promettre des bénéfices monétaires, qu'ils soient explicites ou non. Enfin, nos données permettent d'observer que les partis ont de plus en plus tendance à faire des promesses qui ne font aucune référence à la dimension monétaire : ces propositions s'accroissent par un facteur de trois à chaque élection, tandis que celles dont les bénéfices sont non explicites augmentent seulement de 50\% par élection depuis 1970; on observe que 12 des 15 propositions des programmes de 1960, 1962, 1966 et 1970 font allusion à des bénéfices monétaires alors que seulement 49 des 129 propositions de 1970 à 1981 y font explicitement référence. L'accroissement du nombre (relatif et absolu) des promesses d'interventions s'adressant aux personnes âgées s'accompagne donc d'une réduction de la proportion de celles-ci comportant des bénéfices à caractère monétaire.

Par ailleurs, la place assez modeste qu'elles y occupent pourrait s'expliquer par les coûts très importants engendrés par un nombre restreint de promesses. On a tenté d'évaluer cette question en examinant les informations contenues dans les programmes au sujet des impacts monétaires de leurs promesses. Ils sont assez peu loquaces sur cette question. En effet, 84 des 141 propositions s'adressant aux personnes âgées n'y font aucune référence. D'autre part, l'U.N. se distingue des autres partis en y faisant référence de façon précise à une 
occasion, en 1966, et à nouveau en 1981 en faisant cette fois référence à des coûts monétaires non explicites. Le programme du P.L.Q. de 1960 fait mention de coûts non explicites en ce qui concerne la création d'un fonds de retraite auquel contribueront les employeurs et les employés. Autrement dit, une seule des cent quarante-et-une propositions comporte une référence explicite à ces unités de coûts monétaires et à leur quantité, tandis que deux autres y font expressément état, mais sans en préciser l'ampleur. Enfin, les propositions faisant une référence implicite, c'est-à-dire des promesses de bénéfices monétaires dont on ignore les coûts, totalisent 51 des 141 propositions d'interventions. Les programmes des élections de 1970 et 1973 se distinguent des précédents car, à partir de ce moment, les partis ont une propension de plus en plus forte à ne faire aucune mention à quelque type de coûts que ce soit, qui se traduit, depuis 1970, par le triplement, d'une élection à l'autre, du nombre de propositions de ce type, alors que le nombre de promesses d'action qui comportent des coûts implicites ne fait que doubler.

Il ne faudrait toutefois pas conclure que les promesses d'interventions concernant spécifiquement les personnes âgées n'entraînent pas de coûts importants. Le silence des programmes sur cette question s'explique mieux en référence à leur objectif : un parti qui cherche à maximiser les votes tire avantage à donner le plus de visibilité possible aux bénéfices de ses promesses d'action, tout en en dissimulant les coûts le mieux possible.

\section{b) La divisibilité des bénéfices}

Les bénéfices que véhiculent les promesses d'action des partis peuvent toutefois revêtir beaucoup de tangibilité, tout en ne faisant aucune référence à des avantages monétaires. Aussi a-t-on tenté de déterminer dans quelle mesure les bénéfices promis aux personnes âgées étaient divisibles ou non. Une proposition d'intervention comporte des bénéfices divisibles si les bénéfices promis constituent une somme définie qui peut être fractionnée entre des destinataires. La promesse de construire des centres d'accueil ou des logements qui leur sont réservés constitue un exemple de bénéfice divisible, tandis que la promesse de promouvoir une plus grande humanisation des soins constitue un cas où il est impossible d'établir la somme totale des bénéfices promis.

Le quart des promesses comporte des bénéfices divisibles, alors que $60 \%$ sont indivisibles, le reste ne contenant pas suffisamment d'informations pour les classifier dans l'une ou l'autre catégorie. 
Par contre, même si les propositions qui comportent des bénéfices indivisibles représentent en moyenne 6 propositions sur 10 , les données de notre étude indiquent que si le P.Q. est celui qui a le plus tendance à promettre des bénéfices divisibles, la moyenne mobile montre aussi que c'est celui dont les promesses comportent des bénéfices indivisibles qui s'accroissent le plus rapidement. L'augmentation des bénéfices divisibles se multiplie par un facteur de trois, de 1973 à 1976, alors que celle des indivisibles quadruple, au cours de la même période.

L'examen de la tendance lissée indique aussi que, depuis 1970, le nombre de propositions ne comportant pas suffisamment d'informations pour déterminer leur degré de divisibilité s'accroît par un facteur de deux, d'une période électorale à l'autre, alors qu'au même moment l'augmentation du nombre de bénéfices divisibles triple et celui des indivisibles quadruple.

L'examen des déviations autour de la tendance lissée livre, elle aussi, des résultats intéressants. Celles qui sont les plus élevées concernent les programmes de 1976, dont les bénéfices sont indivisibles, mais surtout les promesses de 1976, dont l'information est insuffisante pour déterminer le degré de divisibilité. L'ampleur de ces déviations confirme d'une autre façon la tendance des partis à offrir de plus en plus de bénéfices indivisibles ou des bénéfices trop imprécis pour en mesurer la divisibilité.

Bref, si l'on évalue le degré de tangibilité des bénéfices par leur degré de divisibilité, il faut conclure qu'environ le quart seulement prend un caractère tangible.

\section{c) Le degré de congestion des bénéfices}

Le caractère plus ou moins tangible des bénéfices se définit, non seulement en rapport à leur divisibilité, mais aussi suivant leur degré de congestion : autrement dit, au-delà d'un certain seuil, la consommation d'une personne réduit la quantité de bénéfices qui peut être retirée au même moment par d'autres individus; il s'agit donc de bénéfices épuisables par opposition à ceux inépuisables, jugés non susceptibles de congestion. Le quart des promesses d'action comporte ce type de bénéfices alors que près de $60 \%$ n'en contiennent pas. Le reste des propositions, soit $15 \%$, est classé dans la catégorie non applicable en raison de l'insuffisance d'informations explicites sur la nature du bénéfice offert.

Les partis se distinguent assez nettement les uns des autres. L'U.N. est celui qui offre le moins de bénéfices susceptibles de congestion 
alors que le P.Q. est celui qui a le plus tendance à en promettre. L'examen de la moyenne mobile indique aussi que si tous tendent davantage à promettre des bénéfices non susceptibles de congestion plutôt qu'épuisables, cette tendance est beaucoup plus forte chez les péquistes que chez les libéraux, les unionistes se classant encore ici en troisième position. Enfin, une proportion croissante des propositions d'interventions ne contient pas suffisamment d'informations explicites sur la nature des bénéfices pour permettre de déterminer leur degré de congestion. Les libéraux arrivent ici en premier avec $19 \%$ de toutes leurs promesses d'action, les péquistes se classant deuxième avec $13 \%$ et l'U.N. arrivant en dernier lieu avec $12 \%$.

Bref, les bénéfices promis dans les programmes d'action tendent à être de plus en plus intangibles, comportant une proportion croissante de propositions dont les bénéfices sont non susceptibles de congestion, avec une proportion croissante de propositions qui ne contient pas suffisamment d'informations explicites pour pouvoir déterminer le degré de congestion des bénéfices.

\section{d) Les critères d'éligibilité aux bénéfices}

Dans quelle mesure l'éligibilité aux bénéfices promis aux personnes âgées dépend-elle d'autres critères que l'âge? On peut répondre à cette question en examinant les critères d'exclusion des bénéfices. Toutes les promesses d'interventions s'adressant aux personnes âgées offrent des bénéfices d'exclusion facile puisqu'elles comportent au minimum une règle d'éligibilité : l'âge. Il faut toutefois noter que près de $15 \%$ de propositions contiennent des règles d'exclusion à caractère discrétionnaire, c'est-à-dire que le critère d'éligibilité à un bénéfice n'est pas fonction de règles normatives explicites. Ce fut le cas, notamment en 1966, lorsque le P.L.Q. promit l'adoption d'une loi générale d'assistance sociale qui engloberait l'assistance aux personnes âgées dans le but de leur dispenser une assistance financière en fonction de leurs besoins réels. On rencontrait le même type de propositions, en 1981, quand le P.Q. promit que les centres d'accueil hébergeraient les personnes âgées qui ont vraiment besoin de soins. L'examen des moyennes mobiles indique que ce type de propositions a eu tendance à diminuer lentement en nombre chez le P.L.Q. et, inversement, à augmenter lentement du côté du P.Q.

D'autre part, un peu plus de $20 \%$ des promesses faites aux personnes âgées comportent des bénéfices d'exclusion facile de type réglementé, c'est-à-dire des propositions dont le critère d'éligibilité est 
fonction de règles normatives explicites. À titre d'illustration, citons le programme du P.Q. de 1981 lorsqu'il promet aux travailleurs qui touchent le salaire minimum une rente correspondant à $100 \%$ du salaire brut. L'examen des moyennes mobiles indique que ce type de promesses d'action a tendance à augmenter beaucoup plus rapidement dans les programmes du P.Q. que dans ceux des autres partis.

Enfin, il faut aussi observer que près de $65 \%$ de toutes les promesses d'interventions comportent d'autres bénéfices d'exclusion facile, c'est-à-dire dont le critère d'éligibilité n'est explicitement ni de type réglementé ni de type discrétionnaire. C'est le cas lorsqu'en 1976 le P.Q. garantit aux personnes âgées le transport en commun gratuit en milieu urbain. Ce type de propositions hybrides a tendance à augmenter plus rapidement que les deux autres chez tous les partis, et cette propension est beaucoup plus forte chez les péquistes que du côté des libéraux.

L'éligibilité aux bénéfices des promesses d'action peut aussi être considérée sous l'angle du statut des bénéficiaires dans le système du marché privé. Les propositions d'intervention comportent-elles des bénéfices pour les détenteurs de facteurs de production ou pour les consommateurs? Plus concrètement, on les a distinguées suivant qu'elles visent les individus à titre de détenteurs du facteur force de travail ou à titre de consommateurs de biens et services. Ainsi, une proposition qui vise à abaisser l'âge de la retraite comporte un bénéfice concentré sur le facteur de production force de travail tandis qu'une qui vise à augmenter les loisirs, le logement et les services de santé en constitue une dont les bénéfices sont diffus. Les données recueillies indiquent qu'un peu plus du quart des promesses d'action concernent des bénéfices concentrés. Le P.Q. et le P.L.Q. ont tous deux tendance à accroître leurs promesses d'action visant les travailleurs âgés. L'ampleur de la déviation autour de la moyenne mobile indique clairement que les programmes du P.Q. et du P.L.Q. de 1976 marquent un point de rupture à partir duquel ces deux partis, mais particulièrement le P.Q., tendent à accroître rapidement le nombre de leurs propositions d'interventions pour cette clientèle particulière.

L'examen des moyennes mobiles montre aussi que les partis ont tendance à accroître le nombre de propositions dont les bénéfices visent les personnes âgées dans des rôles de consommateurs. Le P.Q. arrive, ici aussi, en première position, suivi du P.L.Q. puis, loin derrière, de l'U.N. Il faut toutefois souligner une légère tendance pour le P.Q. et le P.L.Q. à accroître plus rapidement, depuis 1973, la proportion de celles qui comportent des bénéfices concentrés que celles qui contiennent des bénéfices diffus, les partis ayant promis des bénéfices 
concentrés dans plus de trente-quatre des propositions d'action soumises en 1981.

Bref, l'âge ne constitue pas souvent le seul critère d'éligibilité. Les principaux critères reliés à l'âge sont le besoin évalué de façon discrétionnaire, le besoin évalué à l'aide de règles normatives et, finalement, le statut dans le système de marché : détenteur de facteurs de production ou consommateur. Dans l'ensemble, l'accès aux bénéfices est de plus en plus réglementé tout en visant de plus en plus les travailleurs plutôt que les consommateurs.

\section{e) Le degré de précision dans l'évaluation des bénéfices et des coûts}

Finalement, on s'est aussi interrogé sur le degré de précision des bénéfices et des coûts des promesses d'action des partis. Un bénéfice ou un coût est considéré comme facilement mesurable s'il fait simultanément référence à une unité de mesure et à une quantité, par exemple la construction de $\mathbf{1 0 0 0}$ habitations à loyer modique pour les personnes âgées; s'il ne contient pas d'informations sur ces deux critères, il est considéré comme difficilement mesurable. L'exemple typique d'un bénéfice difficile à mesurer est le cas où un parti promet aux personnes âgées d'humaniser les services de santé. Les propositions d'interventions gouvernementales contenues dans les programmes des partis comportent des bénéfices difficiles à mesurer plus de neuf fois sur dix depuis 1960, totalisant jusqu'à 95\% de celles du P.L.Q. et $86 \%$ chez les péquistes.

D'autre part, si l'on excepte une promesse faite par l'U.N. en 1966, les partis ne font jamais mention de coûts qui seraient faciles à mesurer. Ceux-ci sont donc aussi difficiles à mesurer en 1981 qu'en 1960. Par ailleurs, ils sont peu enclins à indiquer s'ils seront supportés par les détenteurs de facteurs de production ou par les consommateurs puisqu'ils font explicitement référence à des coûts concentrés dans six cas et de coûts diffus à une seule occasion.

Au total, force est de conclure que les partis sont peu précis à l'égard des bénéfices et des coûts engendrés par les promesses d'action qu'ils font aux personnes âgées.

\section{Interprétation des résultats}

Le poids des propositions d'interventions s'adressant spécifiquement aux personnes âgées totalise environ le tiers du poids que ces 
personnes représentent dans le corps électoral et l'analyse montre que les bénéfices sont largement symboliques :

- explicitement monétaires pour moins de 10\% des propositions;

- divisibles dans le quart des cas;

- susceptibles de congestion pour un quart des promesses d'action;

- l'accès comporte des règles d'exclusion à caractère discrétionnaire dans $15 \%$ des cas et $20 \%$ dans celui des règles d'exclusion à caractère réglementé ;

- les trois quart des promesses faites aux personnes âgées les visent à titre de consommateurs de services ;

- les bénéfices promis sont difficiles à mesurer, neuf fois sur dix.

D'autre part, les programmes des partis ne contiennent pas beaucoup d'informations sur les coûts de leurs promesses :

- ils ne font aucune référence à des coûts monétaires pour plus de $60 \%$ de leurs promesses d'action;

- si l'on fait exception d'une promesse de l'U.N., ils ne fournissent jamais d'informations qui permettent de mesurer facilement les coûts de leurs projets d'interventions.

Les bénéfices des promesses faites aux personnes âgées sont très largement symboliques tandis que les coûts qui en résultent sont à peu près totalement passés sous silence. Ces résultats indiquent que la question de la population âgée constitue un enjeu où les partis politiques se limitent à adopter des positions de principe. Ils estiment vraisemblablement que les réassurances symboliques contenues dans leurs positions de principe seront suffisantes pour que les électrices et les électeurs les associent à une image positive qui maximise les votes auprès des personnes âgées tout en minimisant les coûts que devrait supporter le reste de l'électorat.

Les bénéfices symboliques peuvent toutefois revêtir une importance considérable. D'après Elder et Cobb (1983: 115), ils peuvent constituer les précurseurs nécessaires à la production de bénéfices plus tangibles :

"Les victoires symboliques qui donnent une sanction "officielle" à la cause d'un groupe tendent à légitimer à la fois le groupe concerné et les intérêts représentés. Sans cette légitimité, le groupe ne peut pas espérer exiger un partage des bénéfices tangibles des politiques."

Qu'on le veuille ou non, les partis sont impliqués dans l'allocation de bénéfices symboliques. L'accroissement du nombre et de la proportion de promesses d'interventions à l'endroit des personnes âgées 
signifie qu'ils reconnaissent les besoins et les demandes de cette population comme un enjeu dont le règlement requiert l'intervention de l'État.

L'évolution des promesses s'adressant explicitement aux personnes âgées porte à penser que l'hypothèse d'Elder et Cobb n'est pas sans fondement. En effet, les données sur les programmes des partis montrent que, si au cours des années soixante ils tendent surtout à viser les personnes âgées dans des rôles de consommateurs en leur promettant des bénéfices symboliques, cette tendance s'estompe progressivement au cours des années soixante-dix, les partis tentant alors de plus en plus à les viser comme détenteurs de facteurs de production - en l'occurrence le travail - en leur promettant des bénéfices beaucoup plus tangibles, notamment à l'égard du régime public de rentes et de l'âge de la retraite. Bref, ce renversement progressif de tendances indique que les bénéfices qui leur sont promis, même s'ils sont encore très largement symboliques, tendent à prendre un caractère de plus en plus tangible.

D'autre part, les données des programmes des partis politiques québécois confirment l'hypothèse de Pomper (1980 : 146), qui suppose que, plus la probabilité de perdre une élection est grande, plus le degré de spécificité des promesses est élevé. Si l'on transpose cette relation à la seule compétition pour l'obtention du vote de la population âgée, on note que le parti qui obtient le plus faible pourcentage au sein de ce groupe d'âge, le P.Q., est aussi celui dont les propositions d'action comportent le degré le plus élevé de bénéfices tangibles. II cherche donc à accroître ses appuis électoraux parmi cette population en lui promettant des bénéfices plus précis et plus tangibles que le P.L.Q. et l'U.N. qui, pour leur part, préfèrent ne pas faire de promesses trop précises de peur de perdre les appuis sur lesquels ils peuvent déjà compter. Au-delà de cette compétition pour les votes, on peut toutefois penser que la présence d'un parti qui reçoit moins d'appuis de la part de la population âgée contribue à inciter les autres à accroître le degré de précision de leurs promesses d'action davantage que si les trois bénéficiaient d'appuis électoraux d'importance semblable au sein de cette population.

\section{Conclusion}

Le poids des propositions d'interventions qui concernent explicitement les personnes âgées dans les programmes des partis totalise environ le tiers du poids qu'elles représentent dans le corps électoral. 
L'analyse des 'caractéristiques de ces promesses indique que cette sousreprésentation ne s'explique pas par le coût élevé engendré par les bénéfices offerts, car ceux-ci sont largement symboliques, alors que les coûts sont à peu près totalement dissimulés.

Les données concernant l'ensemble des promesses d'interventions indiquent que les partis sont en train de segmenter l'électorat suivant des groupes cibles de plus plus nombreux. Ce processus de segmentation croissante signifie que des agrégats tels que la population âgée ou les femmes entrent en compétition avec un nombre croissant de groupes. Aussi faut-il s'attendre à ce que la place relative qu'occupent les personnes âgées dans les programmes n'atteigne jamais le poids qu'elles ont dans le corps électoral. De plus, l'ampleur de leur sousreprésentation ne doit pas être trop exagérée puisqu'un peu plus de $50 \%$ de toutes les promesses s'adressent à plus d'un groupe d'âge, ou encore ne contiennent pas suffisamment d'informations pour déterminer le groupe auquel elles s'adressent. Le résultat le plus sûr qui se dégage est que les partis ne jugent pas l'âge comme un critère approprié d'éligibilité aux bénéfices des biens et services produits par la collectivité.

\section{Note}

* Les données quantitatives utilisées dans cet article ont été recueillies dans le cadre d'une recherche plus vaste qui porte sur l'évolution des interventions gouvernementales au Québec, de 1960 à aujourd'hui. Le lecteur intéressé peut obtenir celles concernant les personnes âgées en s'adressant à l'auteur. Cette recherche a été réalisée grâce à une subvention du Conseil de recherches en sciences humaines du Canada. L'auteur tient à remercier $\mathrm{M}^{\mathrm{me}}$ Paule Duchesneau, professionnelle de recherche, sans laquelle ce projet aurait été impossible à mener à terme.

\section{Références bibliographiques}

Austin, C.D. et M.B. LotB, "Why age is relevant in social policy and practice", dans: B.L. Neugarten (éd.), Age or Need? Public Policies for Older People, Beverly Hills (Ca.), Sage, 1982: 263-288.

CoOK, F.M., "Assessing age as an eligibility criterian», dans : B.L. NeugarTeN (éd.), Age or Need? Public Policies for Older People, Beverly Hills (Cal.), Sage, 1982: 171-204.

CRÊTE, J. et R. LANDRY, "Vieillesse québécoise et changement politique ", Anthropologie et sociétés, vol. 6, nº3, 1982: 45-62. 
Del BAyle, J.L., Introduction aux méthodes en sciences sociales, Paris, Privat, 1978.

Downs, A., An Economic Theory of Democracy, New York, Harper \& Row, 1957.

Elder, C.D. et R.W. COBB, The Political Uses of Symbols, New York, Longman, 1983.

Guillemard, A.-M., "The making of old age policy in France: points of debate, issues at stake, underlying social relations", dans : A.-M. GUILLEMARD (éd.), Old Age and the Welfare State, Beverly Hills (Ca.), Sage, 1983: 75-100.

Guillemard, A.-M., "Jalons pour une sociologie des politiques sociales. Le cas de la politique française de la vieillesse", Sociologie et sociétés, vol. $16, \mathrm{n}^{\circ} 2$, 1984 : 119-128.

HAyes, M.T., Lobbyists and Legislators: A Theory of Political Markets, New Brunswick (N.J.), Rutgers University Press, 1981.

HolstI, O.R., Content Analysis for the Social Sciences and the Humanities, Reading (Mass.), Addison-Wesley, 1969.

Hudson, R.B. et R.H. Binstock, "Political systems and aging", dans: R.H. Binstock et E. ShanAs (éds), Handbook of Aging and the Social Sciences, New York, Van Nostrand Reinhold, 1976 : 369-400.

KRIPPENDORFF, K.H., Content Analysis: An Introduction to its Methodology, Beverly Hills (Ca.), Sage, 1980.

KUTZA, E.A. et N.R. ZWEIBEL, "Age as a criterian for focusing public programs", dans: B.L. Neugarten (éd.), Age or Need? Public Policies for Older People, Beverly Hills (Ca.), Sage, 1982: 55-100.

LANDRY, R., Guide d'utilisation de la fiche d'analyse de l'offre de politiques, Québec, Département de science politique, Université Laval, 1983, 50p.

Ostrogorskı, M., Democracy and the Organization of Political Parties, 2 vols., Chicago (III.), Quadrangle, 1964.

POMPER, G.M. et S.S. LeDerMAN, Elections in America : Control and Influence in Democratic Politics, New York, Longman, 1980.

RoberTSON, D., A Theory of Party Competition, New York, Wiley, 1976.

$Z_{A Y}, N$., "Analyse critique des politiques et des institutions québécoises concernant les personnes âgées", Sociologie et sociétés, vol 16, no 2, 1984 : 105-118. 